\title{
THE FORCING OF A PHOTOCHEMICAL AIR QUALITY MODEL WITH ATMOSPHERIC FIELDS SIMULATED BY A REGIONAL CLIMATE MODEL DATA
}

\author{
Mogesh Naidoo*1 and Francois Engelbrecht ${ }^{2}$ \\ ${ }^{1}$ CSIR NRE Atmospheric Modeling, P O Box 17001, Congella, 4013, South Africa, mnaidoo4@csir.co.za \\ ${ }^{2}$ CSIR NRE Atmospheric Modeling, P O Box 395, Pretoria, 0001, South Africa, fengelbrecht@csir.co.za
}

\begin{abstract}
Summary
Within the context of climate change over southern Africa, little is understood about the potential local response of air quality to changes in the larger scale environment. Under future climate forcing, there may be significant changes in the thermodynamic structure of the atmosphere over southern Africa, and in the circulation dynamics of the region. For example, there is evidence that more intense surface inversion layers may occur over the central interior of South Africa in response to the enhanced greenhouse effect (e.g. Engelbrecht et al, 2009). Such changes are likely to influence the future transport and chemistry of air pollutants over the region. The complexity in which climate change may affect regional air quality is evident.
\end{abstract}

Sophisticated numerical models are required to describe such complexity, and need to take into account all meteorological and emission changes, as well as apply relevant dispersion and chemistry to solving variation in pollutant concentration. The capacity to force atmospheric chemistry models with the output of regional climate models exists within CSIR NRE in the form of the photochemical air quality model CAMx forced by the MM5 regional climate model. This is useful for retrospective studies in air quality however the aspect of future climate forcing on air pollution is not addressed. This paper describes the development of new mode lling capacity suitable for the simulation of photochemistry over southern Africa under both current and future anthropogenic forcing. The model CAMx was nested within the output of the regional climate model CCAM, which is applied at the NRE to obtain detailed projections of regional climate change. This new configuration may potentially be applied for photochemistry modelling at all time-scales, but the emphasis is on the very long integrations suitable to describe photochemistry characteristics over southern Africa at the climatechange time scale.

Here, the new CCAM-CAMx configuration was tested by simulating a selected previously modelled ozone episode, serving to demonstrate the capability of incorporating CCAM data into CAMx as well as investigating how well the new system performs. Results show the importance of preparing appropriate cloud and precipitation data from the CCAM output, as well as a need for finer vertical resolutions in CCAM. In general, CCAM may be appropriately used to force CAMx, illustrating the potential of the new system to simulate photochemistry and air quality over southern Africa under conditions of current and future anthropogenic forcing.

\section{Introduction}

Within the context of climate change over southern Africa, little is understood about the potential response of local air quality to changes in the larger scale environment. Under future climate forcing, there may be significant changes in the thermodynamic structure of the atmosphere over southern Africa, and in the circulation dynamics of the region. For example, there is evidence that more intense surface inversion layers may occur over the central interior of South Africa in response to the enhanced greenhouse effect (e.g. Engelbrecht et al, 2009). Such changes are likely to influence the future transport and chemistry of air pollutants over the region. Climate change may further impact air quality by affecting natural emissions, through the changes in land use and temperature, and by inducing changes in 
anthropogenic emissions through modifying energy needs (e.g. Katragkou et al, 2009). The complexity through which climate change may affect regional air quality is evident.

Sophisticated numerical models are required to describe such complexity. In order to simulate the variations in air pollution concentration that may occur in response to enhanced anthropogenic forcing all meteorological and emission changes need to be considered within the modelling system, in addition to the relevant dispersion and chemistry. The capacity to force atmospheric chemistry models with the output of regional climate models exists within CSIR Natural Resources and Environment (NRE), in the form of a photochemical air quality model, CAMx (Comprehensive Air quality Model with eXtensions), forced by the MM5 regional climate model. Although this capacity is useful for retrospective studies of air quality under present-day climatological forcing, the aspect of future climate forcing on air pollution is not addressed. However, the Conformal-Cubic Atmospheric Model (CCAM), which is applied at the NRE to obtain detailed projections of regional climate change, may be used to provide CAMx with future climate scenarios. This paper describes the development and assessment of a new modelling capacity suitable for the simulation of photochemistry over southern Africa under both current and future anthropogenic forcing. Here a previous photochemical modelling study, focused on the ozone forming potential within the Highveld region, using the MM5/CAMx system (Naidoo, 2009) will be used for the CAMx set up, emissions inventory and comparison (with the new CCAM/CAMx system). That is, an air quality simulation using the MM5 driven CAMx is compared to a similar simulation in which CCAM was used to force CAMx. This procedure tests the suitability of incorporating CCAM meteorological data into a photochemical model such as CAMx, and facilitates a comparison of the CCAM-CAMx system against the well-tested pairing of MM5 and CAMx.

\section{The MM5/CAMx system}

CAMx is a photochemical dispersion model that is suitable for the integrated assessment of gaseous and particulate air pollution. The model allows for integrated "one-atmosphere" (signifying interaction between pollutants) assessments of gaseous and particulate air pollution (ozone, PM2.5, PM10, air toxics) over many scales ranging from sub-urban to continental. It is designed to unify all of the technical features required of "state-of-the-science" air quality models into a single system (Environ, 2008). Since 1996, CAMx has been employed extensively throughout the U.S by local, state, regional, and federal government agencies, academic and research institutions, as well as private consultants for regulatory assessments and general research. It is one of only four photochemical models recommended by the U.S E.P.A (USEPA, 2008). The U.S. E.P.A has approved the use of CAMx for numerous ozone and particulate matter for State Implementation Plans throughout the U.S, and has used this model to evaluate regional mitigation strategies.

Input for CAMx is comprised primarily of spatially and temporally resolved meteorological and emissions data. A common practice is to provide CAMx with meteorological data via a numerical atmospheric model. A model that is often used internationally for this purpose is the Penn State/NCAR Meso-scale Meteorological Model (MM5v3) e.g. (Jung et al., 2004; Emery et al., 2004; Lei et al., 2008). The system has also been applied within the southern African context as part of the Cross Border Air Pollution Impact Assessment, CAPIA (Zunckel et al., 2006).

In addition to emissions and meteorological data, CAMx requires initial and boundary conditions for any (should data be available) emission and pollutant to be modelled. Column ozone/haze/albedo data is necessary, from which photolysis rates are derived. For the MM5/CAMx run used in this paper, column ozone/haze/albedo data from the Ozone Monitoring Instrument (OMI) on NASA's Aura satellite was used. Photolysis rates were derived from this using a pseudo-spherical two-stream delta-Eddington radiative transfer scheme applied via the TUV model, which is developed at the University Corporation for Atmospheric Research (UCAR). The photolysis rates are necessary for driving modelled photochemical reactions and are specific to the chemical mechanisms chosen by the modeller. This CAMx run utilized the Carbon Bond 4 Mechanism 3. Initial and boundary conditions for CAMx are spatially invariant by default, as is the case here, though modellers may develop their own preprocessors and methodology to incorporate varying gridded data. The Global Atmospheric Watch station at Cape Point served as data source for boundary conditions and initializing CAMx for ozone and methane (daily average), while NOx and speciated VOC were initialized with advised values from Environ and data from Brunke et al (2001). Land surface variables such as topography and land type are generally taken from the meteorological model, in this case MM5, which used USGS $4 \mathrm{~km}$ resolution surface data. MM5 was run at a $36 \mathrm{~km}$ resolution and then interpolated to $12 \mathrm{~km}$ resolution for CAMx (Figure 1) by the "mm5camx" pre-processor developed by Environ.

This pre-processor is necessary for preparing meteorological data for CAMx.

MM5 was initialized with the NCEP FNL (Final) Operational Global Analysis data, which are on 
$1.0 \times 1.0$ degree grids and are available at six hourly intervals. The vertical structure consisted of 14 levels, with the lowest level located at a height of about $7 \mathrm{~m}$ above the surface of Earth, with model top at about $15000 \mathrm{~m}$.

The emissions inventory was developed during a previous research project, aimed at understanding ozone dynamics over the Highveld region, particularly contributions from power generation and a synthetic fuels facility in Secunda. However, due to the complex nature of ozone formation, an attempt at including all possible emissions sources was made. Pollutants included in the inventory are $\mathrm{SO}_{2}, \mathrm{NOx}, \mathrm{CO}, \mathrm{NMVOC}, \mathrm{NH}_{3}$ and PM. The spatial extent covers all of South Africa at a resolution of $12 \mathrm{~km}$ (see the CAMx domain shown in Figure 1). Emissions were processed and formatted for CAMx using the Emissions Pre-Processor System (EPS). All VOC and PM speciation profiles for the inventory were derived from the U.S E.P.A Speciation Database (U.S E.P.A, 2006).

The emissions inventory contains the following source categories:

- Residential - Emissions from domestic fuel burning

- Transportation - Emissions from road vehicles, diesel trains and airport ground vehicles

- Large Industry - Emissions from Sasol, Eskom and refineries

- Small Industry - Emissions from smaller more disperse industry

- Biogenic - Emissions from vegetation and soil

\section{The CCAM/CAMx system}

CCAM is a global climate model which is formulated on a quasi-uniform grid (McGregor, 2008). In stretched/variable resolution mode, it may be used at the regional scale (by applying higher resolution over areas of interest). An advantage of this approach to regional modelling,, as opposed to limited-area modelling, is that it provides great flexibility for dynamic downscaling from any global model, essentially requiring only sea-surface temperatures and, optionally, far-field winds from the host model (e.g. Engelbrect et al, 2009). Thus the need for lateral boundary conditions, and the errors that occur in association with wave propagation across these boundaries, are avoided. This makes CCAM particularly useful for both global and regional scale climate change simulations.

It may be noted that limited-area models, such as MM5 or WRF, may in principle also be applied to downscale the climate change scenarios derived from global atmospheric models, thereby providing the necessary output to force CAMx. However, the great computational efficiency of the CCAM code, its flexibility in downscaling output from various global models, and the existing modelling infrastructure that exists around this model at CSIR, has rendered it the model of choice to force CAMx.

The process of preparing meteorological data for use in CAMx has been vastly simplified by the model developers, through applications that process meteorological model data and create the necessary CAMx input. Applications exist for the MM5 ("mm5camx") and WRF ("wrfcamx") model output. Prior to this research, no such application existed for CCAM. Use of CCAM within CAMx is unique to this study and an application, suitably called "ccamcamx", was developed to prepare the required CAMx input from CCAM model output. CCAM is able to provide a majority of the required fields, though some have to be derived. There are also some variables that can not be derived using the existing CCAM model code, thereby inducing some extensions of the existing CCAM code. A summary of fields used during this study is listed in Table 1.

Table 1: Summary of fields used to force CAMx

\begin{tabular}{|l|c|c|}
\hline CAMx requirement & $\begin{array}{l}\text { Direct from } \\
\text { CCAM }\end{array}$ & $\begin{array}{l}\text { Derived from } \\
\text { CCAM }\end{array}$ \\
\hline Land-use & $\checkmark$ & \\
\hline Topography & & \\
\hline 3D layer interface height & & $\checkmark$ \\
\hline 3D layer average pressure & $\checkmark$ & \\
\hline 3D layer average UWind & $\checkmark$ & \\
\hline 3D layer average VWind & $\checkmark$ & \\
\hline 2D temperature & $\checkmark$ & \\
\hline 2D rain precipitation & $\checkmark$ & \\
\hline 3D layer average temperature & & \\
\hline 3D layer average water vapour & & $\checkmark$ \\
\hline 3D layer average cloud water content & $\checkmark$ \\
\hline 3D layer average ice water content & $\checkmark$ \\
\hline
\end{tabular}




\begin{tabular}{|l|l|l|}
\hline 3D layer average rain water content & & \\
\hline 3D layer average snow water content & & \\
\hline 3D layer average graupel water content & & \\
\hline Column cloud optical depth & & $\checkmark$ \\
\hline 3D layer interface vertical diffusivity & & $\checkmark$ \\
\hline
\end{tabular}

Fields assumed/derived for use in CAMx include:

- 3D height: CCAM provides layer interface geopotential height ("zg"), though in meters above sea level. CAMx requires height at meters above ground level. This requirement was calculated by subtracting "zg" from CCAM surface height ("zs"). The first model level in the vertical is located at a height of about $30 \mathrm{~m}$ above ground level.

- 3D pressure: CCAM simulates the surface pressure ("ps"). This is sufficient information for layer average pressure, as required by CAMx, to be calculated on each of the sigma (normalised pressure) coordinate levels of CCAM.

- 3D cloud water: CCAM does not explicitly provide cloud water content. With respect to water phase variables, CCAM does however provide liquid water ("qlg") and frozen water ("qfg"). For now, it was assumed here that cloud water content be taken from CCAM "qlg".

- 3D ice water: Ice water refers to all water in the ice phase, though should not be confused with ice precipitation (seen as snow or hail). This plays a role in chemistry (thermodynamic and physical) and column optical depth. Here it was assumed that CCAM "qfg" (frozen water) be taken as 3D CAMx ice water.

- Surface rain water: CCAM provides simulations of both convective and non-convective rain at the surface. This aids in determining pollutant deposition and adds to optical depth.

- Cloud optical depth: CAMx requires this variable to determine photolysis rates as a function of available solar radiation. Since the photolysis rates determine rate of photochemical reactions, this variable is vital in modelling ozone formation. Cloud optical depth is derived using algorithms found in the "mm5camx" and "wrfcamx" application code, and is a function of either convective or non-convective precipitating water content (all phases).

- 3D vertical diffusivity: CAMx requires this variable to describe vertical transport between model layers. It is a derived variable, not readily produced by meteorological models. With respect to preparing MM5 or WRF output for use in CAMx, the methodology of O' Brien (1970) is used to determine the vertical diffusivity. This methodology was used to prepare vertical diffusivity from CCAM output. The necessary fields (PBL height, 3D height, 3D temperature, 3D water vapour, 3D $U$ and $V$ wind) are already available from CCAM.

Fields not derivable from CCAM:

- Land-use: This field is necessary for CAMx to determine surface roughness and type, and is used to calculate pollutant deposition rates. CAMx reads in 11 land-use categories. These 11 categories were not readily available from within CCAM (which uses a different classification of land-use), and therefore the MM5 land-use fields are being used for the moment. At a later stage of the CCAM-CAMx development process, the relationship between the CAMx and CCAM land surface categories will be determined.

- 3D rain water: This field is necessary to aid liquid phase chemistry in CAMx and plays a role in wet scavenging and optical depth calculations when preparing meteorological fields. CCAM does not provide this field for each layer (only rain reaching the surface is available as a variable) though it is likely that "qlg" (liquid water) is a combination of cloud water content and precipitating water. For this study, rain water for layers above surface has been left as zero.

- 3D and surface snow water: Similar to rain water, snow affects CAMx chemistry and wet scavenging, as well as assisting in optical depth calculations. CCAM does not provide this field for each layer. It is also possible that CCAM frozen water (qfg) is partially made up of precipitating snow. For this study, snow water has been left as zero, with clarity on how to deduce the variable from the different model levels to be obtained from the CCAM code developers.

- 3D graupel water: Graupel plays a role in CAMx chemistry by providing physical surfaces for reaction and deposition. It also affects optical depth to a large extent. Due to CCAM being predominantly a global or regional forecast model, focus has shifted away from this microscale phenomenon. The adequate description of graupel dynamics thus does not feature in the model output. It is envisaged that ongoing collaborative research between NRE AMSi and the CSIRO will alleviate this void. For this study 
however, graupel water has been left as zero.

The CCAM model produces output on a conformal-cubic grid coordinate system. CAMx requires data to be on a geographic coordinate system of latitude/longitude, Universal Transverse Mercator (UTM) projection or Lambert Conformal projection. Previous CAMx model runs performed at CSIR were set up on the UTM system. All other model input, including the MM5 land-use, was therefore prepared on the same grid. It was therefore deemed the most convenient to provide CCAM variables on the same grid. Through postprocessing (developed by CSIRO) it is possible to write CCAM output to a lat/lon grid, though no routine exists to write to UTM. It was therefore necessary to interpolate CCAM variables from the lat/lon grid to the UTM grid used by CAMx. This was achieved through a bicubic interpolation routine.

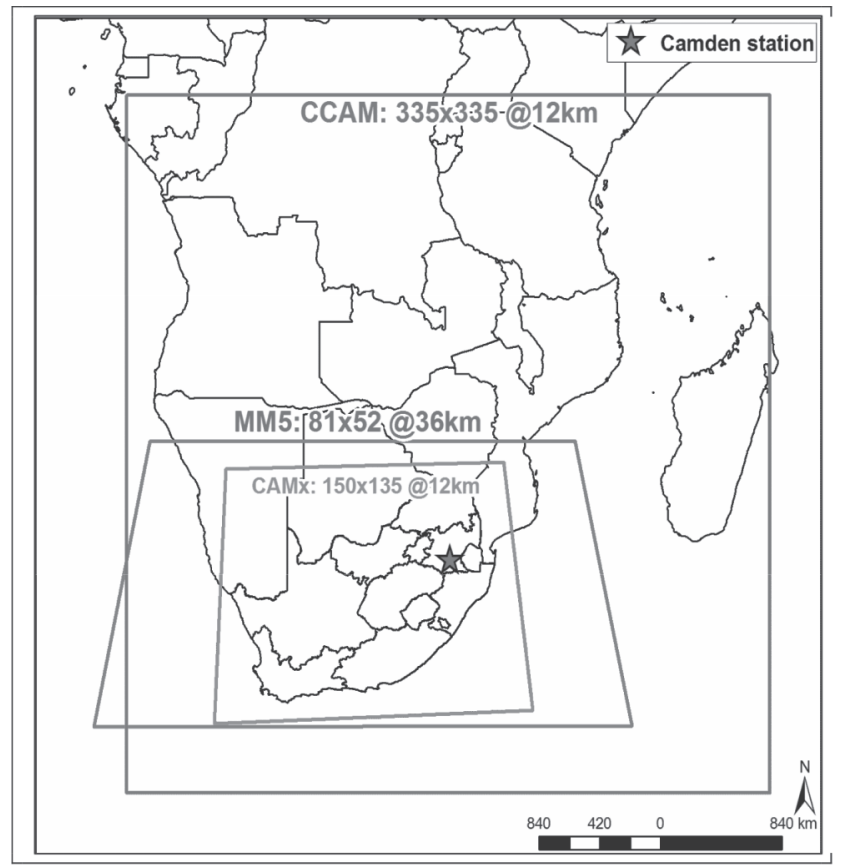

Figure 1: CCAM, MM5, CAMx modelling domain and location of Camden monitoring station

\section{Results}

Seven days $\left(11^{\text {th }}-17^{\text {th }}\right.$ of December 2006) of hourly CCAM output was used to force CAMx. The first of these days served as time for model spin-up. Simulations for the same period were performed earlier using the MM5/CAMx system. The results shown here are comprised of a comparison between the MM5/CAMx and CCAM/CAMx simulated ozone output, as well as a comparison of both model simulations against measured surface ozone data. The measured data is taken from a monitoring station in Camden (Figure 1), thus all comparisons are performed for this location. The choice of monitoring station is based purely on data availability. The analysis represents a test of suitability of CCAM to force CAMx, but only a preliminary indication of the CCAM/CAMx system performance, as not all meteorological scenarios have been accounted for (as discussed earlier). Indeed, the assumptions mentioned in section 3 need to be understood with regard to their effects on CAMx, and taken into account when analysing the simulation results.

The assumptions play different roles in the solution CAMx reaches, with some increasing and others decreasing ozone formation. Ozone production would increase due to more solar radiation reaching the surface from less interference in the CCAM simulations, since 3D rain, snow and graupel were taken as zero (affecting cloud optical depth). In addition, wet scavenging would be at a minimum in upper layers,

leaving more ozone in the gas phase. A decrease in ozone production would arise from the assumption that all liquid water from CCAM (qlg) was taken to be cloud water, thus increasing cloud effects and possibly cloud thickness, leading to less solar radiation available to the surface. Each of these factors may dominate the effects on ozone concentrations, depending on the prevailing meteorological conditions.

For this modelling period, the CCAM/CAMx system is seen to give considerably higher optical depth than the MM5/CAMx system. Figure 2 shows the column integrated optical depth for both modelling systems.

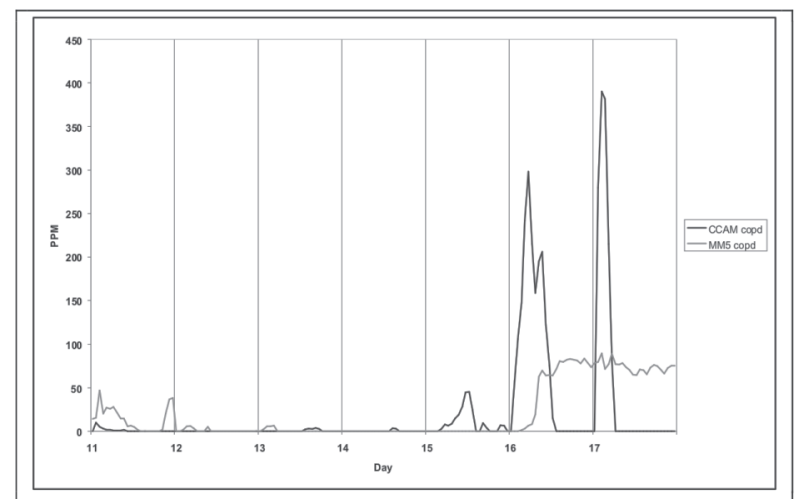

Figure 2: CCAM/CAMx and MM5/CAMx system derived column integrated optical depth

The higher optical depth values in the CCAM/CAMx system are expected, as it overestimates cloud water content - due to the assumption that all liquid water be taken as cloud water in the CCAM/CAMx system. The optical depth peaks present in the CCAM/CAMx system may also be a result of this assumption. However, the difference in meteorological model resolution may also play a role as the higher resolution CCAM run (at $12 \mathrm{~km}$ ) should give greater ability to simulate extreme values than the lower resolution MM5 run. The lack of 3D precipitation output in CCAM has not become a factor for this study as meteorological 
conditions show no rain or snow for both CCAM and MM5.

A higher optical depth results in less solar radiation reaching the surface, and thus lower photolysis rates. This, however, does not exclusively result in lower ozone production. Ozone formation may be due to various chemical mechanisms, each with their own peak reaction rates. Although the photolytic reactions play a large role, their function in the ultimate result of ozone formation is far from linear. Having said this, a comparison of surface ozone at the Camden site, between the CCAM/CAMx and MM5/CAMx system (Figure 3), shows lower ozone values corresponding roughly with periods of enhanced derived optical depth.

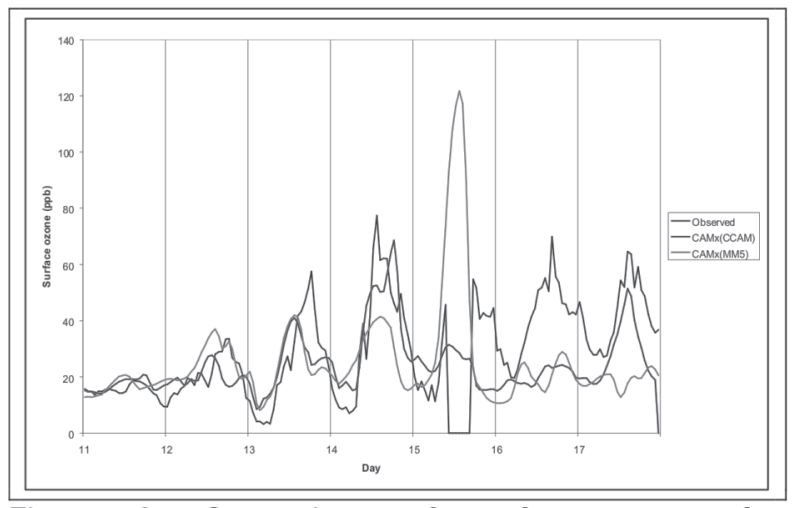

Figure 3: Comparison of surface ozone from CCAM/CAMx, MM5/CAMx systems and monitoring station at Camden

This phenomenon is particularly pronounced during day 16, when optical depth derived from CCAM data starts increasing. The CCAM/CAMx system simulated that ozone did not peak significantly during midday on the $16^{\text {th }}$, although a pronounced peak was observed. Day 17 does show a simulated ozone peak that compares well to the observed peak, but only some time after the highest optical depth was simulated (this occurred at 6am and dropped abruptly thereafter). It is also apparent that the MM5/CAMx system does not present an ozone peak during $16^{\text {th }}$ and 17 th, and this may also be seen as a result of too high optical depth. Indeed, the MM5/CAMx system is seen to under-predict at most days when compared to CCAM/CAMx ozone and observations. When compared to observed data, the CCAM/CAMx shows better performance than the MM5/CAMx system, for days when optical depth did not play a role., The MM5/CAMx system consistently underpredicted ozone values, except for day 12 and 15. The $15^{\text {th }}$ is interesting as MM5/CAMx system predicts a pronounced peak, while observed data is unavailable. CCAM/CAMx does not predict this peak. Looking at Figure 2, one might correlate this to optical depth, with MM5/CAMx giving no optical depth and CCAM/CAMx showing approximately
$50 \mathrm{ppm}$ on the $15^{\text {th }}$. The total lack of optical depth from the MM5/CAMx system, combined with CCAM/CAMx showing a limited though present value, may contribute, at least partially, to CCAM/CAMx system not predicting an as prominent peak as the MM5/CAMx system. Indeed the opposite may have occurred on the $17^{\text {th }}$, when MM5/CAMx optical depth limits ozone formation.

\section{Conclusion}

A CCAM/CAMx photochemical modelling system is currently being developed with the eventual aim of predicting the response of air quality over southern Africa under scenarios of climate change. This requires appropriate assimilation of CCAM data as input into CAMx. A pre-processor, "ccamcamx", was developed to achieve this task. Here, an analysis of suitability and performance of the CCAM/CAMx system is given for an initial iteration of the "ccamcamx" application.

While CCAM is able to provide CAMx with most meteorological parameters, 3D rain, snow, graupel and land use remain unavailable. This may reduce the impact of wet scavenging in the upper layers and show various effects on the derived optical depth. The assumption that all liquid water in CCAM be taken as cloud water in CAMx has led to an overestimation in optical depth. The lack of wet scavenging has not been illustrated since there was no significant precipitation during the modelling period used. These limitations withstanding, the CCAM/CAMx system performs admirably in comparison to MM5/CAMx and observed data. Further research directed at obtaining 3D precipitation and land-use from CCAM will be of great benefit to developing a modelling framework for ascertaining the impact of climate change on air quality.

\section{References:}

Brunke, E.G., Labuschagne, C., and Scheel, H.E., 2001. Trace gas variations at Cape Point, South Africa, during May 1997 following a regional biomass burning episode. Atmospheric Environment. 35. 777-786.

Emery C., Jia Y., Kemball -Cook S., Mansell G., Lau S. and Yarwood G. (20 04). Modeling an August 1322, 1999 ozone episode in the Dallas/Forth Worth area. Prepared for Texas Commission on Environmental Quality. Environ International Corporation.

Engelbrecht F.A., McGregor J.L. and Engelbrecht C.J. (2009). Dynamics of the confo rmal-cubic atmospheric model projected climate-change signal over southern Africa. International Journal of Climatology. 29, $1013-1033$. 
ENVIRON., 2008. User's guide to the Comprehensive Air Quality Model with Extensions (CAMx) v4.50, ENVIRON International Corporation, 101 Rowland Way, Novato, CA 94945.

Jung L., Kim M. and John K. (2004). Sensitivity of modeled ozone in a coastal urban airshed. 3rd Annual CMAS Models-3 Users' Conference. October 18-20, 2004 Chapel Hill, NC.

Katragkou E., Zanis P., Tegoul ias I., Melas D., Kruger B.C., Huszar P., Halenka T., and Rauscher S. (2009). Decadal regional air quality simulations over Europe in present climate: near surface ozone sensitivity to external meteorological forcing. Atmospheric Chemistry and Physics Discussions. 9, $10675-10710$.

Lei W., Zavala M., de Foy B., Volkamer R. and Molina L.T. (2008). Characterizing ozone production and response under different meteorological conditions in Mexico City. Atmospheric Chemistry and Physics Discussions. 8, $12053-12079$.

McGregor J.L., Dix M.R. (2008). An updated description of the Conformal-Cubic Atmospheric Model. In Hamilton K. and Ohfuchi W. (eds). High resolution numerical modelling of the atmosphere and ocean. Springer, New York. Chapter 4, 51 -75.

O'Brien, JJ. (1970). A note on the vertical structure of the eddy exchange coefficient in the planetary boundary layer. J Atmos Sci 27:1213-1215.

U.S E.P.A., 2008. Photochemical Modelling. Technology Transfer Network

Support Center for Regulatory Atmospheric Modeling.

http://www.epa.gov/scram001/photochemicalindex. htm, accessed July 2009.

\section{U.S E.P.A., 2006. SPECIATE 4.0: SPECIATION \\ DATABASE DEVELOPMENT \\ DOCUMENTATION. Office of Research and \\ Development U.S. Environmental Protection Agency. EPA/600/R -06/161.}

Zunckel, M., Koosailee, A., Yarwood, G., Maure, G., Venjonoka, K., van Tienhoven, A.M., Otter, L., 2006. Modelled Surface Ozone Over Southern Africa During the Cross Border Air Pollution Impact Assessment Project. Environmental Modelling \& Software. 21, 7. 911 - 924. 